\title{
Comparison of Indoor Propagation Models at 2.4GHz for 5G Wireless Mobile Networks using SDN Simulator
}

\author{
Haru Alhassan ${ }^{1,2}$, Raed Abdulhameed ${ }^{1,4}$, Mohammed J. Ngala ${ }^{3}$, Chidi Diugwu ${ }^{2}$ \\ \{haru@ncc.gov.ng, mjngala@ heatoned.co.uk, cdiugwu@ncc.gov.ng \} \\ Faculty of Engineering and Informatics University of Bradford U.K. ${ }^{1}$, \\ Nigerian Communications Commission Abuja Nigeria ${ }^{2}$, \\ Heaton Education Bradford U.K. ${ }^{3}$ \\ Information and Communication Eng. Department, Basrah University College of Science and \\ Technology, Basrah 24001, Iraq ${ }^{4}$
}

\begin{abstract}
In this paper, a comparison on performance of indoor radio propagation models for $5 \mathrm{G}$ wireless networks is presented. The $5 \mathrm{G}$ wireless networks will be marked with movement of large volume of data and support for newer applications that require much high bandwidth (IoT, Ultra-Reliable and low latency Communications, Industrial Automations, Robotics, Drones and Virtual Reality to mention just few). There is going to be serious challenge in meeting the RF signal coverage for indoor environments. Although there is no single RF propagation model that is suitable for all indoor environments, a number of popular indoor propagation models were compared using Mininet-Wifi Simulator at $2.4 \mathrm{GHz}$. ITU-T model is recommended because of its superior performance over the other three propagation models. The channel characteristics was analyzed based on Received Signal Strength Indicator (RSSI).
\end{abstract}

Keywords: Transmit Power, Path Loss, RSSI, SINR, Mininet-Wifi.

\section{Introduction}

In the recent years, data traffic has tremendously increased due to availability of smartphones and increase of the use of Social Media Sites by all ages especially middle ages. The mobile traffic volume is expected to grow tremendously in the next few years as a result envisaged Internet of Things (IoT) applications and services. It is expected that, over 50 billion mobile devices will be connected to the network by 2020, and ever increasing necessity of access and share data, anywhere and anytime [1], [2]. The figure is expected to rise to over 70 billion mobile devices by 2025 . The rapid increase in the number of connected devices necessitated the need to increase the capacity of the existing network structure [3]. There is also the need for large number of Micro and Pico cells for the improvement of the signal strength and quality in the indoor environment. This necessitated channel modelling particularly for indoors environment which offers opportunities for further investigation of the effects that environmental conditions (building architecture, construction materials and number of floors) can have of the channel characteristics [4].

Based on applications of attractive services such as virtual reality [5] that depends on the 4G LTE technologies to enable the new 5G radio. This also supports the Ultra-Reliable and 
Low-Latency Communication (URLLC) for short latency services that should apply new more cells for coverage.

A number of $5 \mathrm{G}$ channel propagation models were studied in literature, this includes propagation model at $20 \mathrm{GHz}$ and $30 \mathrm{GHz}$ for indoors and outdoors. Path Loss Model was investigated in at $40 \mathrm{GHz}$ in [3] for LOS and NLOS scenarios. In [5] and [6] propagation measurements were conducted at $6-38 \mathrm{GHz}$, and for mmwave in [7] that also covering the 2$73.5 \mathrm{GHz}$ bands for indoor office and shopping mall environments. Cluttered channels discussed in [3] that includes more sophisticated models.

To develop and validate accurate model for 5G, an extensive measurement campaign is needed for different environments and environmental conditions. The PL model was detailed carefully in 3D models in [3], and the PL exponent for various channels models in [8], [9]. More details will be stated in the following section detailed of buildings with many floors.

\section{Indoor Propagation Modelling}

There are numerous theoretical and experimental studies of indoor propagation models [38]. Most of the models tend to focus more on small scale fading or inter-floor or loses as a result of obstacles like walls between transmitter and receiver. This study is aimed at developing an indoor propagation model using Mininet-Wifi, SDN Simulator.

Indoor radio propagation is dominated by the same mechanisms as outdoor: reflection, diffraction, and scattering [10]. However, conditions are much more variable, because opening and closing of doors can make great difference in signal strength. Partition losses for same floor and multiple floor losses can also exist which greatly affect the signal strength. The work in [10] tabulate the average Floor Attenuation Factors (FAF) for one to four floors in two office building. Also tabulated are the PL exponents and standard deviation measured in different buildings.

\subsection{Log-Distance Path Loss Model}

The average large scale PL can be illustrated from [10],

$$
P L(d B)=P L(d o)+10 n \log \left(\frac{d}{d o}\right)+X \sigma
$$

Where $\mathrm{n}$ and $\mathrm{X} \sigma$ are PL exponent and shadowing factor respectively. A good set of data to define various mobile environments can be found from [10-11].

\subsection{Log Normal Shadowing}

Based on the shadowing the propagation path could be stated as Log-Normal Distribution [10]. This PL can be simply given by: 


$$
P L(d B)=P L(d o)+10 n \log \left(\frac{d}{d o}\right)+X \sigma
$$

Where $\mathrm{X} \sigma$ is a zero-mean Gaussian distributed random variable with the standard deviation $\sigma . \mathrm{N}$ is the PL exponent for the channel [12].

\subsection{ITU-R Indoor Propagation Model}

The ITU-R indoor PL is formally expressed by the following:

$$
P L(d)[d B]=20 \log (f)+N \log (d)+P f(n)-28
$$

Where:

$P L=$ The total path loss $(\mathrm{dB})$.

$f=$ Operating frequency $(\mathrm{MHz})$

$d=$ Distance $(\mathrm{m})$.

$N=$ The distance power loss coefficient.

$n=$ Number of floors between the $\mathrm{T}$ and $\mathrm{R}$.

$P f(n)=$ The floor loss penetration factor.

Data from the ITU-R was summarized in Table 1.

Table 1. Distance Power Loss Factor

\begin{tabular}{|l|l|l|l|}
\hline $\begin{array}{l}\text { Frequency } \\
\text { Band GHz }\end{array}$ & $\begin{array}{l}\text { Residential } \\
\text { Area }\end{array}$ & Office Area & $\begin{array}{l}\text { Commercial } \\
\text { Area }\end{array}$ \\
\hline 0.900 & NA & 33 & 20 \\
\hline $1.2-1.3$ & NA & 32 & 22 \\
\hline $1.8-2.0$ & 28 & 30 & 22 \\
\hline 4 & NA & 28 & 22 \\
\hline 5.2 & $30 A p t, 28$ Hous & 31 & NA \\
\hline 5.8 & NA & 24 & NA \\
\hline 6.0 & NA & 22 & 17 \\
\hline
\end{tabular}

Loss in floor is practically measured to perform the whole loss channel when many floors considered. Some data provided by ITU-R are stated in Table 2.

Table 2. Floor Penetration Loss Factor

\begin{tabular}{|l|l|l|l|l|}
\hline $\begin{array}{l}\text { Freq } \\
\text { Band GHz }\end{array}$ & $\begin{array}{l}\text { No. of } \\
\text { Floors }\end{array}$ & $\begin{array}{l}\text { Reside } \\
\text { Area }\end{array}$ & Office Area & $\begin{array}{l}\text { Commercial } \\
\text { al } \\
\text { Area }\end{array}$ \\
\hline 0.900 & 1 & NA & 9 & NA \\
\hline 0.900 & 2 & NA & 19 & NA \\
\hline 0.900 & 3 & NA & 24 & NA \\
\hline $1.8-2.0$ & $n$ & $4 n$ & $15+4(n-1)$ & $6+3(n-1)$ \\
\hline 5.2 & 1 & NA & 16 & NA \\
\hline 5.8 & 1 & NA & $22(1 \mathrm{fl}), 28(2 \mathrm{fl})$ & NA \\
\hline
\end{tabular}




\subsection{Attenuation Factor}

Channel propagation in building was studied for example in [13] for which the PL attenuation was varied between two buildings based on log-distance model as predicted by the following:

$$
P L(d)[d B]=P L\left(d_{0}\right)[d B]+10 n_{S F} \log \left(\frac{d}{d_{0}}\right)+F A F[d B]
$$

Where $n_{S F}$ represent the exponent value for the "same floor" [10]. For multiple floors the equation (4) becomes:

$$
P L(d B)=P L\left(d_{0}\right)+10 n_{M F} \log \left(\frac{d}{d_{0}}\right)
$$

Where $n_{M F}$ is the PL exponent for multiple floors. Based on the results of [14] equation (4) can be modified as:

$$
P L(d)[d B]=P L\left(d_{0}\right)[d B]+20 \log \left(\frac{d}{d_{0}}\right)+\alpha d+F A F[d B]
$$

Where $\alpha$ is constant defines the channel attenuation, measured by $\mathrm{dB} / \mathrm{m}$. Some values of $\alpha$ for some frequencies are given in Table 4 [14].

Table 4. Details of $\alpha$ values.

\begin{tabular}{|l|l|l|}
\hline $\begin{array}{l}\text { Location for the } \\
\text { Building }\end{array}$ & Frequency & $\alpha$-Attenuation $(\mathrm{dB} / \mathrm{m})$ \\
\hline $1: 4$ story & $850 \mathrm{MHz}$ & 0.620 \\
\hline & $1.7 \mathrm{GHz}$ & 0.570 \\
\hline & $4.0 \mathrm{GHz}$ & 0.470 \\
\hline $2: 2$ story & $850 \mathrm{MHz}$ & 0.480 \\
\hline & $1.7 \mathrm{GHz}$ & 0.350 \\
\hline & $4.0 \mathrm{GHz}$ & 0.230 \\
\hline
\end{tabular}

\section{Simulation Setup}

There were quite good number of software to support the network performance such as that discussed through the network emulator used in [13], SDN [14], and the Mininet emulator [15] that provides various experiments and tests to define the network quality services.

The experimental setup consists of small cell and three nodes, one acting an access point and other as users devices. In this scenario one cell is used in order to avoid interferences from the nearby cells. Three nodes were used as access points and connect to the access point 
sartorially at $120^{\circ}$ to each other. One of the nodes was made to move radially away from the access point at constant velocity.

In the scenarios the transmission power of the cell was fixed as against in [4] in which the cell power was being increased and the nodes were kept at constant position, it was only the position of one of the node that changes by moving away radially from the access point. This was done for the node at various points from the cell. The RSSI is used to illustrate the position of the node for the entire network using various indoor propagation models.

\section{Results and Discussions}

\section{Scenario 1: Tx Power of 15dBm}

Figure 1. illustrate the chart for the PLs for $15 \mathrm{dBm}$ Tx power. The ITU PL model outperforms the other models followed by Friis path loss model (with $s L=6$ ). The least in performance is Log-Distance PL model. It can be seen that, at a distance of $10 \mathrm{~m}$ from the access point the RSSI measured for ITU model was $-35 \mathrm{dBm}$, Friis $-42 \mathrm{dBm}$, Log-Normal $-51 \mathrm{dBm}$ and Log-Distance $-53 \mathrm{dBm}$. At distance of $20 \mathrm{~m}$ from the access point the RSSI measured for ITU model was $-40 \mathrm{dBm}$, Friis $-49 \mathrm{dBm}$, Log-Normal $-59 \mathrm{dBm}$ and Log-Distance $-61 \mathrm{dBm}$.

PATH LOSS MODELS PLOT

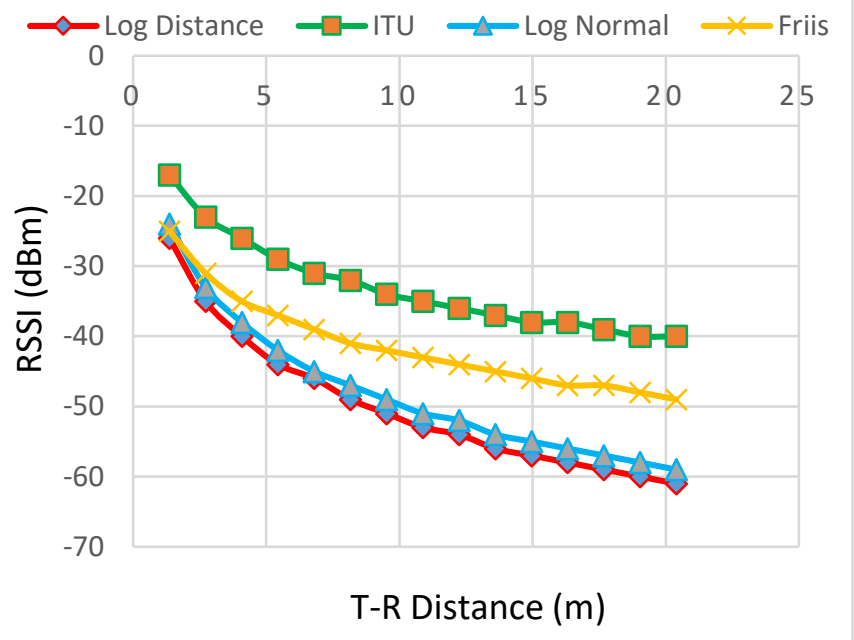

Figure 1. RSSI versus T-R Distance Plot

Figure 2. is the Log plot for the same simulation result. It can be seen that for the Log plot the graphs are almost straight lines with the ITU model having the least steep gradient. This also indicated that the ITU model out performs the other indoor models. 


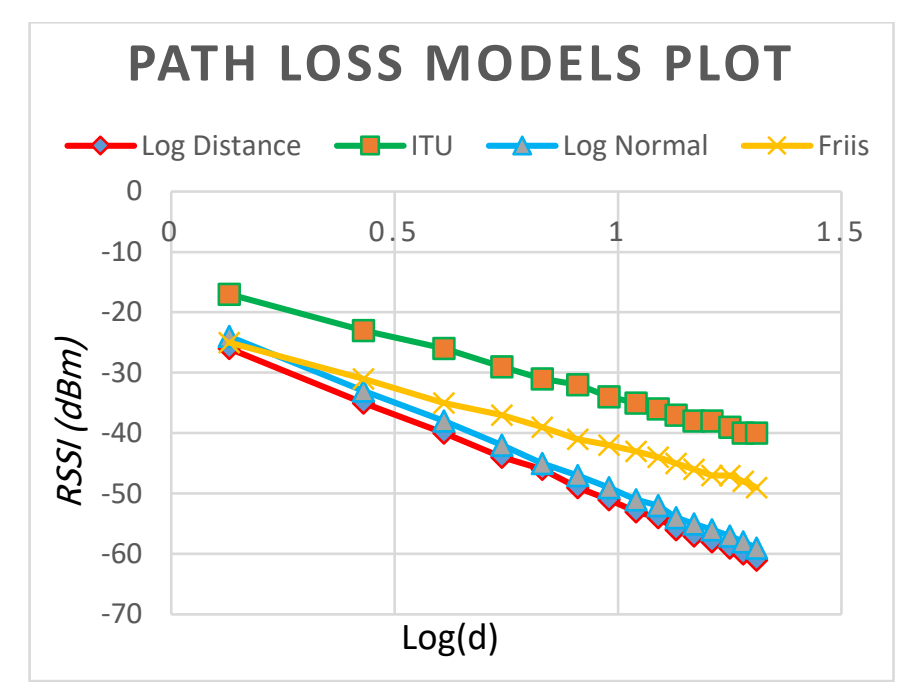

Figure 2. RSSI versus Log of T-R Distance Plot

\section{Scenario 2: Tx Power of 20dBm}

Figure 3. illustrate the chart for RSSI performance of the PT models for 20dBm Tx power. The ITU PL model outperforms the other models followed by Friis path loss model (with sL = 6). The least in performance is the Log-Normal. It can be seen that, at a distance of $10 \mathrm{~m}$ from the access point the RSSI measured for ITU model was $-30 \mathrm{dBm}$, Friis $-38 \mathrm{dBm}$, Log-Normal $47 \mathrm{dBm}$ and Log-Distance $-46 \mathrm{dBm}$. At distance of $20 \mathrm{~m}$ from the access point the RSSI measured for ITU model was $-35 \mathrm{dBm}$, Friis $-44 \mathrm{dBm}$, Log-Normal $-57 \mathrm{dBm}$ and Log-Distance $-56 \mathrm{dBm}$.

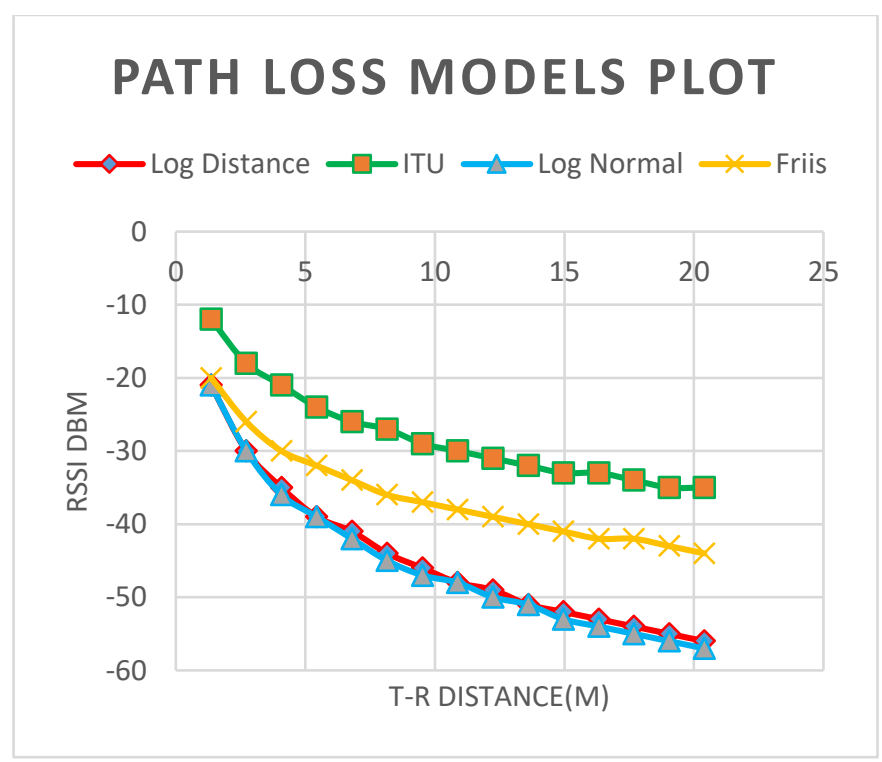

Figure 3. RSSI versus Log of T-R Distance Plot 
Figure 4. is the Log plot for the same simulation result. It can be seen that for the Log plot the graphs are almost straight lines with the ITU model having the least steep gradient. This also indicated that the ITU model out performs the other indoor models.

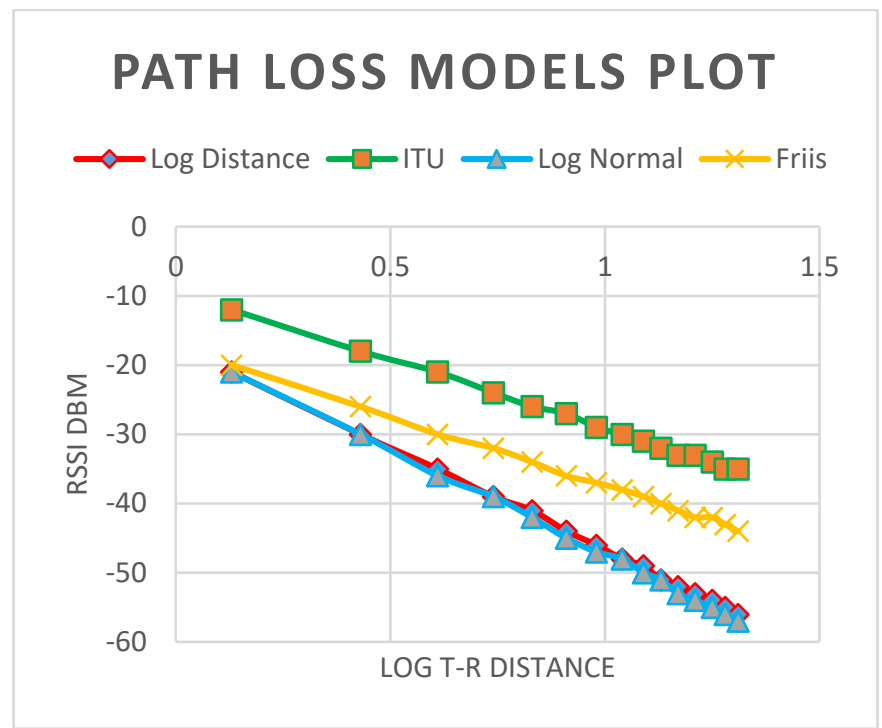

Figure 4. RSSI versus Log of T-R Distance Plot

\section{Scenario 3: Tx Power of 25dBm}

Figure 5. illustrate the PL for $25 \mathrm{dBm}$ Tx power. It shows the ITU PL loss model outperforms the other models followed by Friis path loss model (with $\mathrm{sL}=6$ ). The least in performance is the Log-Normal. It can be seen that, at a distance of $10 \mathrm{~m}$ from the access point the RSSI measured for ITU model was $-25 \mathrm{dBm}$, Friis $-33 \mathrm{dBm}$, Log-Normal $-47 \mathrm{dBm}$ and LogDistance $-43 \mathrm{dBm}$. At distance of $20 \mathrm{~m}$ from the access point the RSSI measured for ITU model was $-30 \mathrm{dBm}$, Friis $-39 \mathrm{dBm}$, Log-Normal $-55 \mathrm{dBm}$ and Log-Distance $-51 \mathrm{dBm}$. 


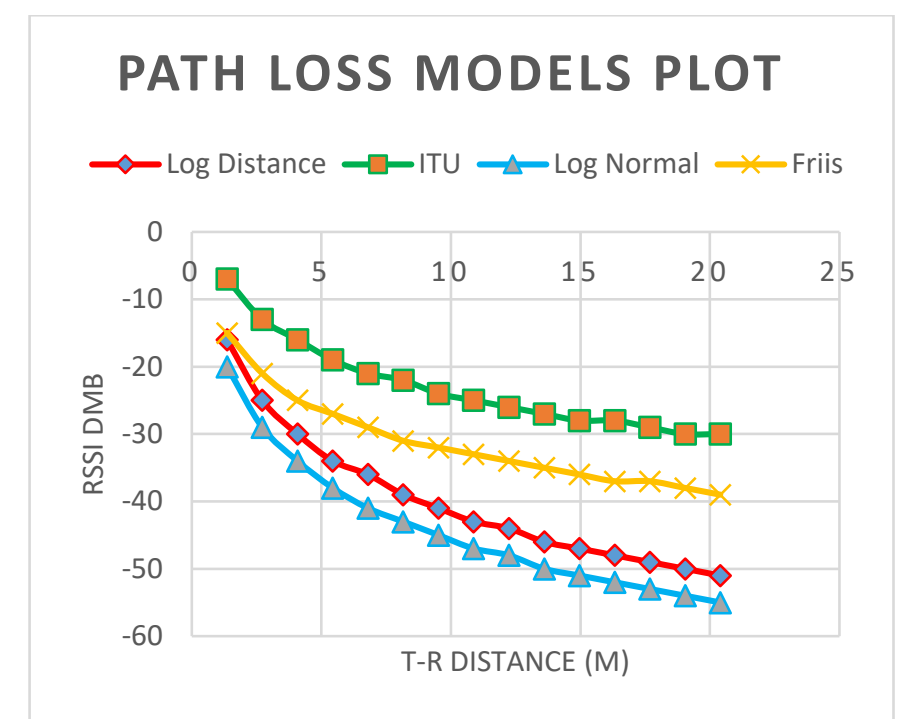

Figure 5. RSSI versus Log of T-R Distance Plot

Figure 6. is the Log plot for the same simulation result. It can be seen that for the Log plot the graphs are almost straight lines with the ITU model having the least steep gradient. This also indicated that the ITU model out performs the other indoor models.

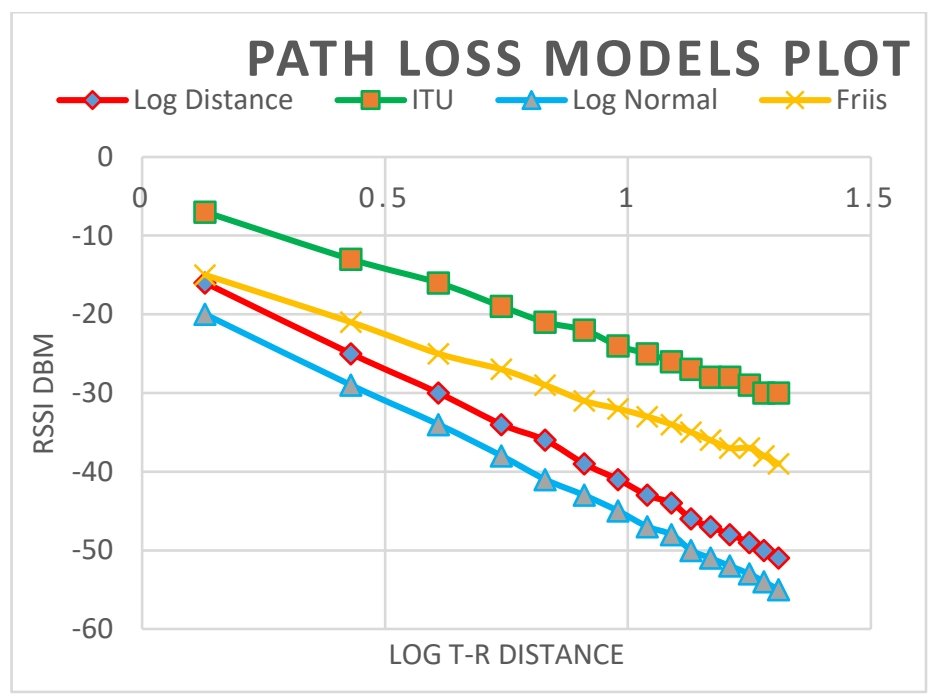

Figure 6. RSSI versus Log of T-R Distance Plot

\section{Scenario 4: Tx Power of 30dBm}

Figure 7. illustrate the chart for PL models for $30 \mathrm{dBm}$ TX power. This shows ITU path loss model also outperforms the other models followed by Friis path loss model (with $\mathrm{sL}=6$ ). The least in performance is Log-Distance path loss model. It can be seen that, at a distance of 
$10 \mathrm{~m}$ from the access point the RSSI measured for ITU model was $-20 \mathrm{dBm}$, Friis $-28 \mathrm{dBm}$, LogNormal $-36 \mathrm{dBm}$ and Log-Distance $-38 \mathrm{dBm}$. At distance of $20 \mathrm{~m}$ from the access point the RSSI measured for ITU model was $-25 \mathrm{dBm}$, Friis $-34 \mathrm{dBm}$, Log-Normal $-44 \mathrm{dBm}$ and Log-Distance $46 \mathrm{dBm}$.

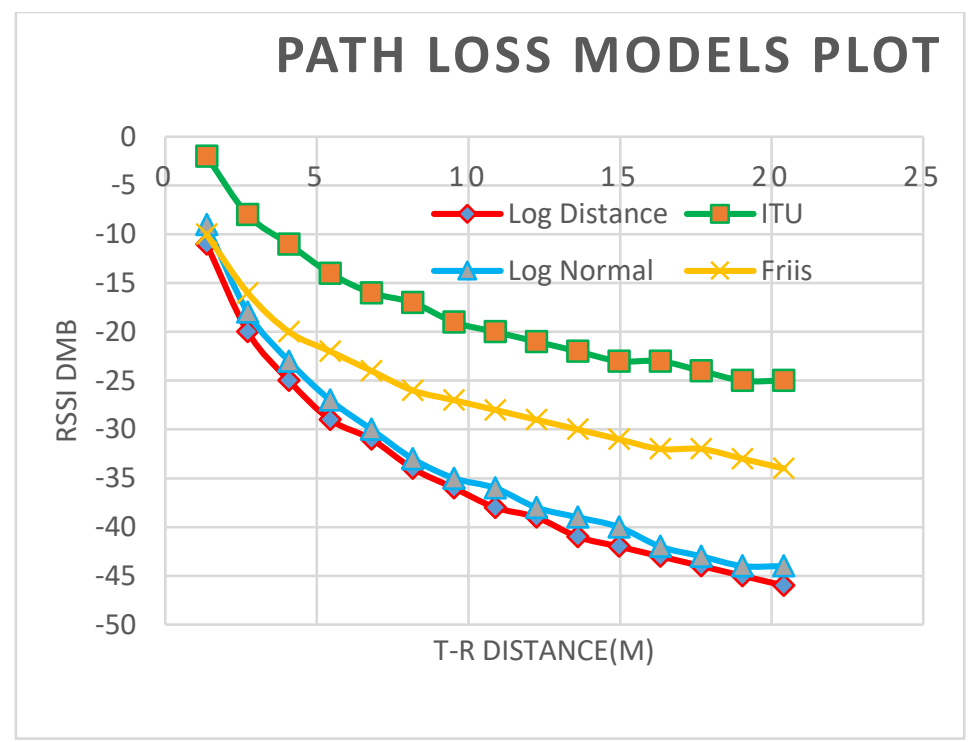

Figure 7. RSSI versus Log of T-R Distance Plot

Figure 8. is the Log plot for the same simulation result. It can be seen that for the Log plot the graphs are almost straight lines with the ITU model also having the least steep gradient. This also indicated that the ITU model out performs the other indoor models.

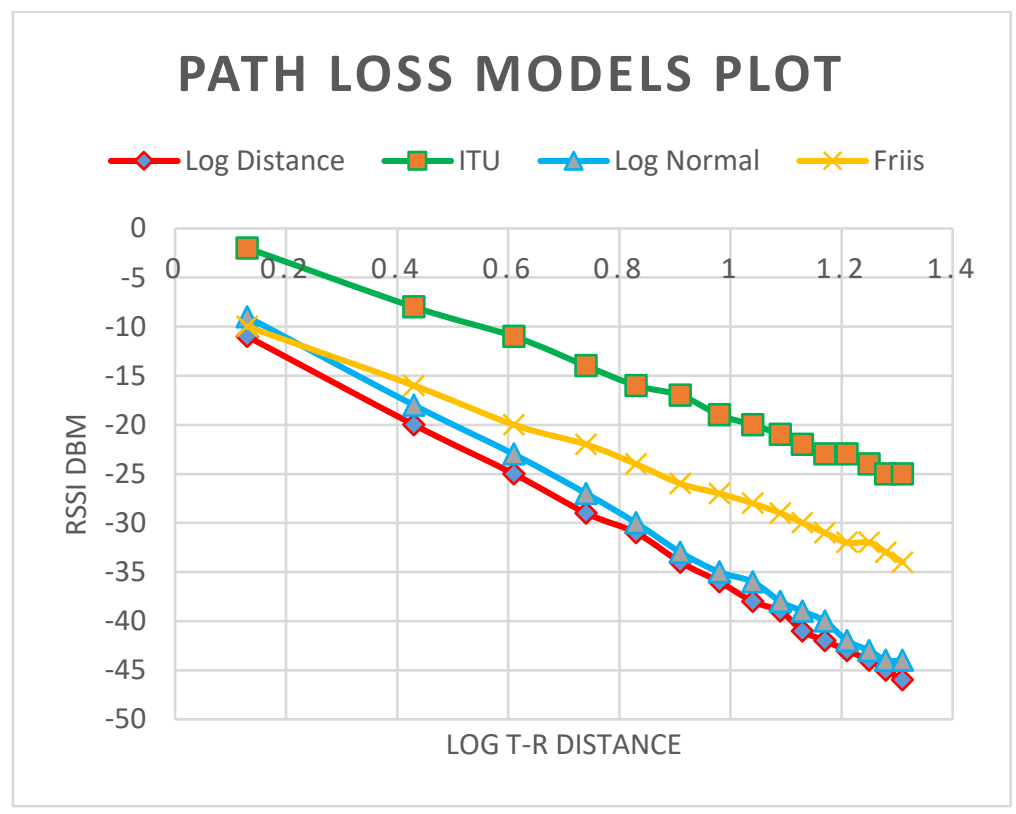


Figure 8. RSSI versus Log of T-R Distance Plot

\section{Conclusions}

In this work, performance of indoor Path Loss Models were compared at $2.4 \mathrm{GHz}$ using Mininet-Wifi Simulator. Mininet-Wifi provide inexpensive platform for testing wireless networks without spending money for building the actual network. The Simulation result will give the actual network performance when deployed. The simulation environment can also provide means for configuration changes and the effects before the deployment of the actual network. The result indicated that the ITU indoor path loss model have superior performance over the other models by being as close as possible to the actual measured result and could be good to further investigate the model in future research. The future research work will focus on SINR, Latency and Throughput performances in addition to RSSI.

\section{References}

[1] A. Osseiran, F. Boccardi, V. Braun, K. Kusume, P. Marsch, M. Maternia, O. Queseth, M. Schellmann, H. Schotten, H. Taoka, H. Tullberg, M. A. Uusitalo, B. Timus and M. Falgren, "Scenarios for $5 \mathrm{G}$ mobile and wireless communications: the vision of the METIS project.," IEEE Communications Magazine, pp. 26-35, May 2014.

[2] A. Gupta and R. K. Jha, "A survey of 5G network: Architecture and Emerging Technologies.," IEEE Access, pp. 1206-1232, 2015.

[3] A. M. Al-Samman, T. Abdul-Rahman, M. H. Azmi, A. Sharaf, Y. Yamada and A. Alhammadi, "Path Loss Model in Indoor Environment at $40 \mathrm{GHz}$ for $5 \mathrm{G}$ Wireless Network," IEEE 14th International Colloquium on Signal Processing and its Applications (CSPA 2018), pp. 7-12, 9-10 March 2018.

[4] T. Akhtar, I. Politis and S. Kotsopoulos, "Wireless Channel Characterisation over Simulations for an indoors Environment at 2.4GHz," Wireless Telecommunications Lab, Patras, Greece, 2018.

[5] Research and Markets, "2018 Leading 5G Applications and Services Research Bundle Research Report," PRNewswire, Dublin, November, 30, 2018.

[6] Al-Samman A. M., Rahman T. A., Azmi H. M., Hindia M. N., Khan I. and Hanafi E., "Statistical Modeling and Characterisation of Experimental mm-Wave Indoor Channels for Future 5G Wireless Communication Networks," PLos One, vol. 11, no. 9, p. 0163034, Sep 2016.

[7] T. A. Thomas, M. Rybakowski, S. Sun, T. S. Rappaport, H. Nguyen and I. Z. Kovacs, "A Prediction Study of Path Loss Models fron $2-73.5 \mathrm{GHz}$ in an Urban-Micro Environment," IEEE 83rd Vehicular Technology Conference (VTC-2016), May 2016.

[8] S. Sun, T. S. Rappaport, T. A. Thomas, A. Gosh, H. C. Nguyen, I. Z. Kovacs, I. Rodriguez, O. Koymen and A. Partyka, "Investigation of Prediction Accuracy, Sensitivity, and Parameter Stability of Large-Scale Propagation Path Loss Models for 5G Wireless Communications," IEEE Trans. Vehicular Technology, vol. 65, no. 5, pp. 2843-2860, 2016. 
[9] A. M. Al-Samman, T. A. Rahman, M. Hadri, I. Khan and T. H. Chua, "Experimental UWB indoor channel charcterization in stationary and mobility scheme," Measurement, vol. 111, pp. 333-339, 2017.

[10] T. S. Rappaport, Wireless Communications Principles and Practice, Upper Saddle River, NJ: Prentice Hall, 1999.

[11] A. Neskovic, N. Neskovic and G. Paunovic, "Modern Approaches in Modelling of Mobile Radio Systems Propagation Environment," IEEE Communications Survey, 2000 .

[12] R. Akl and D. Tummala, "Indoor Propagation Modelling at 2.4GHz for IEEE 802.11 Networks," in the 6th IASTED International Multi-Conference on Wireless and Optical Communications, Banff, AB, Canada, 2006.

[13] F. K. R., "Network Emulation in the Vint/NS Simulator," in 4th IEEE Symposium on Computers and Communications, 1999.

[14] D. Kreutz, F. V. M. Ramos, P. E. P. Verissimo, C. E. Rothenberg, S. Azodolmolky and S. Uhlig, "Software Defined Networking: A comprehensive Survey," IEEE Proceeding, vol. 103, no. 1, 2015.

[15] L. B., H. B. and M. N., "A Network in a Laptop: Rapid prototyping for softwaredefined networks," in 9th ACM SIGCOMM Workshop on Hot Topics in Networks, New York, 2010. 